\title{
Synthesis and Electrical Properties of La-Pr-Mn-O Thin Films and Heterostructures
}

\author{
R. Butkuté $*$ F. Anisimovas, A.K. Oginskis,
}

A. Steikūniené, J. Devenson and B. Vengalis

Semiconductor Physics Institute, A. Goštauto 11, 01108 Vilnius, Lithuania

In this paper we report the results of synthesis and study of both ceramic samples and thin films of electronically doped $\mathrm{La}_{0.7} \mathrm{Pr}_{0.3} \mathrm{MnO}_{3}$ and related heterostructures composed of $\mathrm{La}_{0.7} \mathrm{Pr}_{0.3} \mathrm{MnO}_{3}$ and $p$-type $\mathrm{La}_{0.67} \mathrm{Ca}_{0.33} \mathrm{MnO}_{3}$. The ceramic $\mathrm{La}_{0.7} \mathrm{Pr}_{0.3} \mathrm{MnO}_{3}$ samples were prepared by a conventional solid state reaction technique. Single phase $\mathrm{La}_{0 .}{ }_{7} \mathrm{Pr}_{0.3} \mathrm{MnO}_{3}$ thin films and $\mathrm{La}_{0.7} \mathrm{Pr}_{0.3} \mathrm{MnO}_{3} / \mathrm{La}_{0.67} \mathrm{Ca}_{0.33} \mathrm{MnO}_{3}$ heterostructures were grown on lattice-matched perovskite $\mathrm{NdGaO}_{3}$ substrates by pulsed laser deposition. Electron doping was indicated both for ceramic $\mathrm{La}_{0.7} \mathrm{Pr}_{0.3} \mathrm{MnO}_{3}$ samples and thin films from thermopower data. Both ceramic samples and thin films of $\mathrm{La}_{0.7} \mathrm{Pr}_{0.3} \mathrm{MnO}_{3}$ demonstrated resistivity of about 10 $\mathrm{m} \Omega \mathrm{cm}$ at $300 \mathrm{~K}$ and semiconductor-like resistance vs. temperature behavior with cooling down to $78 \mathrm{~K}$. Meanwhile, the resistance of the $\mathrm{La}_{0.7} \mathrm{Pr}_{0.3} \mathrm{MnO}_{3} / \mathrm{La}_{0.67} \mathrm{Ca}_{0.33} \mathrm{MnO}_{3}$ interface showed an anomalous peak at $185 \mathrm{~K}$. A series of post-deposition annealing experiments demonstrated a crucial role of annealing temperature and ambience on both electrical and magnetic properties of $\mathrm{La}_{0.7} \mathrm{Pr}_{0.3} \mathrm{MnO}_{3}$ material and the heterostructures.

PACS numbers: 75.70.-i, 71.30.+h, 73.50.-h, 75.70.Pa

\section{Introduction}

Perovskite manganites are widely investigated for their huge magnetoresistance and many interesting properties. Hole doping is usually achieved in the $\mathrm{La}_{1-x} \mathrm{~A}_{x} \mathrm{MnO}_{3}$ compounds by substituting a bivalent cation $\mathrm{A}=\mathrm{Ca}, \mathrm{Ba}$, or $\mathrm{Sr}$ at a rare earth site. During the last few years, there was an increasing interest in electronically doped manganites prepared by partial replacing trivalent La by a tetravalent $\mathrm{Ce}, \mathrm{Te}, \mathrm{Sb}, \mathrm{Zr}$ as well as mixed valence $\mathrm{Pr}$ ions [1]. In this case, a mixed-valence $\left(\mathrm{Mn}^{3+}-\mathrm{Mn}^{2+}\right)$ state is expected, and thus one could expect the appearance of electron doping on the manganese site $[2,3]$. Such electron-doped system

${ }^{*}$ corresponding author; e-mail: renata@pfi.lt 
could be very promising for fabrication of $p-n$ homojunctions in novel spintronics devices. Unfortunately, up to now electron-doped manganites are not well examined compared to similar hole-doped systems. Moreover, our previous investigations $[4,5]$ showed a significant change of the $\mathrm{La}_{2 / 3} \mathrm{Ce}_{1 / 3} \mathrm{MnO}_{3}$ film composition making the electron doping questionable.

In this work we systematically investigated synthesis and electrical properties of both ceramic samples and thin films of $\mathrm{La}_{0.7} \mathrm{Pr}_{0.3} \mathrm{MnO}_{3}$ (LPMO). The results of measurements of LPMO and $p$-type $\mathrm{La}_{0.67} \mathrm{Ca}_{0.33} \mathrm{MnO}_{3}$ (LCMO) heterostructures are presented, too.

\section{Experimental details}

The ceramic samples were prepared by a conventional solid state reaction using high purity powdered $\mathrm{La}_{2} \mathrm{O}_{3}, \mathrm{Pr}_{6} \mathrm{O}_{11}$ and $\mathrm{Mn}_{2} \mathrm{O}_{3}$. After being mixed, the powders were heated in air at $950^{\circ} \mathrm{C}$ for $8 \mathrm{~h}$ followed by an intermediate grinding for homogenization, pelletizing and final sintering at $1300^{\circ} \mathrm{C}$ for $8 \mathrm{~h}$. Since the critical temperature value of phase transition to ferromagnetic state of LPMO depends strongly on microstructure and oxygen content, the effects of post-annealing under oxidation and reduction atmospheres on electrical and magnetic properties were examined. The as-prepared ceramic samples were additionally annealed at $1300^{\circ} \mathrm{C}$ either in a flowing $\mathrm{O}_{2}$ or Ar ambience.

A disk-shaped ceramic target with stoichiometric $\mathrm{La}_{0.7} \mathrm{Pr}_{0.3} \mathrm{MnO}_{3}$ composition was used for preparation of thin films by pulsed laser ablation. Thin LPMO films were deposited on monocrystalline $\mathrm{NdGaO}_{3}(100)$ and $\mathrm{MgO}(100)$ substrates. The substrate temperature and oxygen gas pressure was $750^{\circ} \mathrm{C}$ and $25 \mathrm{~Pa}$, respectively. A typical thickness of the films was about $400 \mathrm{~nm}$. After film deposition, the growth chamber was vented with pure oxygen (1 atm) and the films were cooled down slowly to a room temperature. The hole-doped $\mathrm{La}_{0.67} \mathrm{Ca}_{0.33} \mathrm{MnO}_{3}$ film was used for fabrication of $p-n$ structure.

The crystalline structure of the prepared ceramic samples and films was examined by X-ray diffraction measurements using monochromatic $\mathrm{Cu} K_{\alpha}$ radiation. The type of electrical conductivity was identified from thermopower data. Electrical resistance measurements were performed in a temperature range from 78 to $300 \mathrm{~K}$ by a standard four-point-probe configuration and applying dc current of about $10 \mu \mathrm{A}$. The dependence of LCMO/LPMO interface resistance vs. temperature was measured using a three-point-probe method (a current flows through both films in perpendicular direction to the plane). Magnetoresistance (MR) of the samples was measured by applying a magnetic field of $0.3 \mathrm{~T}$.

\section{Results and discussion}

X-ray diffraction (XRD) measurements revealed a single-phase composition of both ceramic samples and thin films. $\Theta-2 \Theta$ XRD spectra demonstrated a polycrystalline quality of thin LPMO films grown directly on $\mathrm{NdGaO}_{3}$ and $\mathrm{MgO}$ 

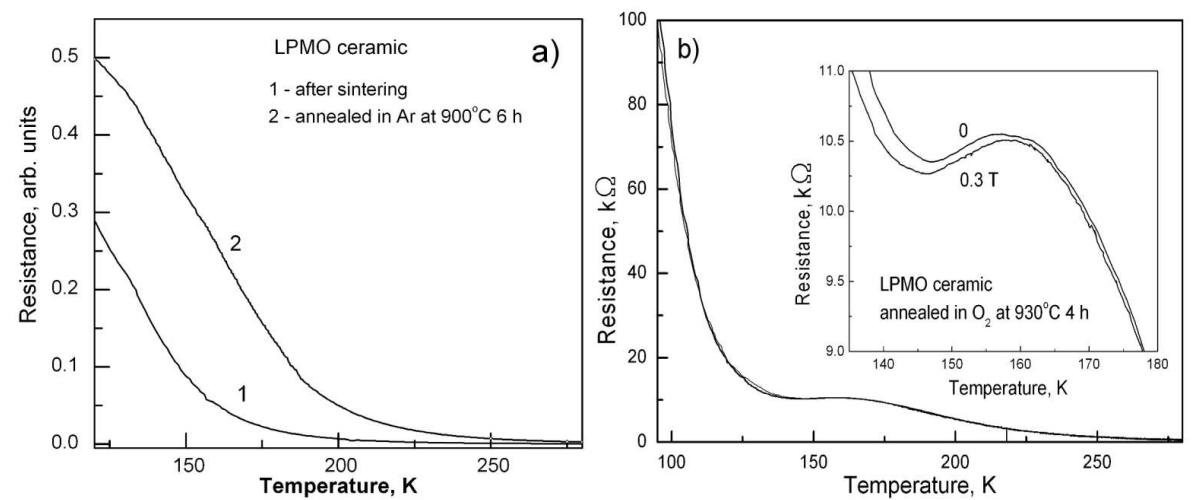

Fig. 1. (a) Resistance versus temperature dependences measured for LPMO ceramics: 1 - after sintering, 2 - after annealing in Ar gas at $900^{\circ} \mathrm{C}$ for $6 \mathrm{~h}$; (b) after annealing in pure oxygen atmosphere at $930^{\circ} \mathrm{C}$ for $4 \mathrm{~h}$. The inset presents behaviors of resistance in the vicinity of the M/I transition under zero and $0.3 \mathrm{~T}$ magnetic fields.

substrates as well as on LCMO film. The thermopower data showed $n$-type of conductivity of both ceramic samples and thin films annealed in various ambiences. The results of electrical measurements of LPMO ceramics are presented in Fig. 1a, b. The curve 1 (Fig. 1a) demonstrating a semiconductor-like behavior of resistance was measured for the sintered ceramic samples in zero magnetic field. The resistance of the samples remains almost unchanged after post-annealing either in Ar gas or vacuum (curve 2, Fig. 1a). Meanwhile, after additional annealing in a flowing oxygen gas at $930^{\circ} \mathrm{C}$ for $4 \mathrm{~h}$, a small peak appeared in the $R(T)$ curve in the vicinity of $165 \mathrm{~K}$ (see Fig. 1b). It is clearly seen from the inset in Fig. 1b that the sample resistance decreased under applied magnetic field of $0.3 \mathrm{~T}$. This allows one to predict from the results that the saturation of the samples by oxygen and homogenization of the ceramic material took place during high temperature annealing. This probably initiates the metal/insulator $(\mathrm{M} / \mathrm{I})$ phase transition and the resulting MR effect. In comparison to the $T_{\mathrm{c}}$ value characteristic of LPMO material $(210 \mathrm{~K})$, the obtained $T_{\mathrm{MI}}$ values are significantly shifted to the region of lower temperatures. Our observations are consistent to similar results for ceramic samples presented in [1]. We explain the lower transition temperature assuming the presence of granular crystalline structure of the samples.

Figure 2 demonstrates the dependences of interface resistance on temperature measured by three-point-probe method for the LPMO/LCMO heterostructure grown on $\mathrm{NdGaO}_{3}$ substrate (curve 1). In order to analyze the data we plotted the $R(T)$ dependences measured for LCMO film. It can be seen from the plot that the LCMO film exhibits a relatively sharp M/I transition at $125 \mathrm{~K}$ with MR effect of about $45 \%$. Though the resistance of LPMO film drastically increases with temperature decreasing down to $78 \mathrm{~K}$, and no transition to a metallic state is observable (there is no curve in the picture), the interface of LPMO/LCMO 


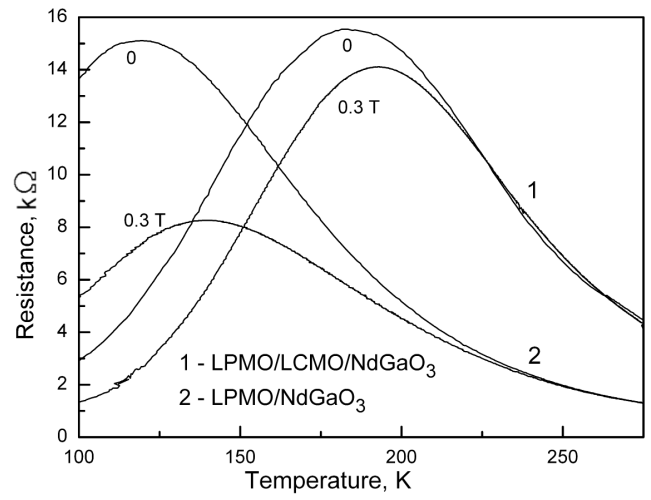

Fig. 2. Dependence of resistance on temperature measured for LPMO/LCMO heterostructure grown on monocrystalline $\mathrm{NdGaO}_{3}$ - curve 1 ; curve 2 presents the behavior of LCMO film. Both curves were measured at 0 and $0.3 \mathrm{~T}$ magnetic field.

heterostructure exhibited an anomalous peak. The peak at $185 \mathrm{~K}$ in the interface resistance could be related to the formation of interlayer between LPMO and LCMO with composition, oxygen content, and crystalline structure close to the bulk material.

\section{Summary}

The results of synthesis of ceramic samples and thin films of $\mathrm{La}_{0.7} \mathrm{Pr}_{0.3} \mathrm{MnO}_{3}$ and heterostructures composed of LPMO and $p$-type LCMO are presented in this work. XRD measurements revealed a single-phase composition of both ceramic samples and thin films. $\Theta-2 \Theta$ XRD spectra demonstrated the polycrystalline quality of thin LPMO films. The thermopower data confirmed $n$-type of conductivity of LPMO ceramic samples and thin films. Electrical measurements demonstrated the resistivity of about $10 \mathrm{~m} \Omega \mathrm{cm}$ at $300 \mathrm{~K}$ and semiconductor-like resistance vs. temperature behavior with cooling down to $78 \mathrm{~K}$ sintered ceramics and thin films of $\mathrm{La}_{0.7} \mathrm{Pr}_{0.3} \mathrm{MnO}_{3}$. Post-annealing experiments demonstrated a crucial role of annealing ambience: after annealing in oxygen gas at $930^{\circ} \mathrm{C}$ for $4 \mathrm{~h}, \mathrm{M} / \mathrm{I}$ transition appears at $165 \mathrm{~K}$. We demonstrated the first attempt to transfer the material from ceramic bulk to thin film and fabricate the $p-n$ heterostructure. The nature of the peak at $185 \mathrm{~K}$ in the temperature dependence of interface resistance of LPMO/LCMO is not clear to the end. One of reasons might be the formation of interlayer between LPMO and LCMO with higher crystalline quality and close to the optimum oxygen content.

\section{Acknowledgments}

This work was partially supported by Swedish Institute within Visby program. 


\section{References}

[1] P. Duan, Z. Chen, S. Dai, Y. Zhou, H. Lu, K. Jin, B. Cheng, Appl. Phys. Lett. 84, 4741 (2004).

[2] P. Mandal, S. Das, Phys. Rev. B 56, 15073 (1997).

[3] C. Mitra, P. Raychaudhuri, G. Kobernik, K. Dorr, K.H. Muller, L. Schultz, R. Pinto, Appl. Phys. Lett. 79, 2408 (2001).

[4] R. Butkutè, J. Devenson, M.A. Rosa, A.K. Oginskis, F. Anisimovas, A. Vailionis, B. Vengalis, Lithuanian J. Phys. 46, 89 (2006).

[5] B. Vengalis, J. Devenson, K. Šliužienè, R. Butkute, M.A. Rosa, V. Lisauskas, A.K. Oginskis, F. Anisimovas, Thin Solid Films 515, 599 (2006). 